\title{
Design of knee support device based on four-bar linkage and hydraulic artificial muscle
}

\author{
Sarin Kittisares ${ }^{1 *}$, Hiroyuki Nabae ${ }^{1}$, Gen Endo ${ }^{1}$, Koichi Suzumori ${ }^{1}$ and Ryo Sakurai ${ }^{2}$
}

\begin{abstract}
One of the main challenges for the elderly is insufficient lower limb strength during sit-to-stand movement, which may be improved by supporting the joint externally. Existing lower extremity exoskeletons use perfect revolute joints as knee joints, which do not match with human joint biomechanics. They also require a complex control system to produce the required torque at the corresponding joint angle. In this study, a knee support device using four-bar joint mechanism and hydraulic artificial muscle (HAM) was designed. A previously proposed four-bar linkage joint was modified to accommodate the HAM. In addition, the Angled Bar was proposed to exploit HAM's force-contraction relationship to generate the desired torque at the corresponding angle only by applying constant hydraulic pressure without the use of a complex control system. The device was able to generate a maximum output of $126.55 \mathrm{Nm}$ torque at $100^{\circ}$ knee joint angle during loading and $70.69 \mathrm{Nm}$ torque at $100^{\circ}$ during unloading at $3 \mathrm{MPa}$ pressure. The root-mean-square error of the knee extension torque curve was $13.01 \mathrm{Nm}$. Experiment with a healthy participant showed significant reduction in muscle activity with the assist from the device. The maximum processed EMG signal with and without assist were 52.10 and $20.93 \mu \mathrm{V}$, respectively.
\end{abstract}

Keywords: Hydraulic artificial muscle, Exoskeleton, Knee joint extension movement

\section{Introduction}

Sit-to-stand (STS) is one of the most important actions for independent living. On average, a free-living adult performs 60 STS actions per day [1,2]. However, STS is also a major problem for older individuals; most falls suffered by the elderly occur while performing an STS transfer $[3,4]$. Moreover, falls are one of the main causes of accidental injuries and death for older people $[5,6]$.

Physical weakness of the elderly is the main cause of falling accidents [6]. Adequate lower extremity strength is a more significant factor for successful STS [7], than balance control [8]. Therefore, existing assistive devices aim to provide mechanical assistance to support STS motion [9-14].

\footnotetext{
*Correspondence: kittisares.s.aa@m.titech.ac.jp

${ }^{1}$ Department of Mechanical Engineering, Tokyo Institute of Technology,

2-12-1 Ookayama, Meguro-ku, Tokyo 152-8550, Japan

Full list of author information is available at the end of the article
}

Current STS assist devices can be roughly divided into three main categories: manual, powered, and exoskeletons. Manual assistive devices, including transfer poles, assist rails, and handles, do not directly provide mechanical assistance but depend on the user's upper extremities to contribute additional force to support the STS motion. Therefore, a lack of upper body strength will limit this type of devices' usefulness. Powered assistive devices, on the other hand, provide powered assistance to support STS action of the user. This type of device includes powered seat $[9,10]$ and stand assist power lift. While these devices are effective on assisting STS transfer, they are often large and not easily moved between locations. Furthermore, their applicability is limited to STS support.

Exoskeletons, on the other hand, are worn by the patients and have much better mobility than powered type. Moreover, they provide direct torque assist to the respective joints, and therefore are potentially beneficial to other applications such as power assist. For STS assist, 
exoskeletons can be beneficial to both patients who are unable to deliver torque at all, and patients who can provide limited lower-limb torque. For the former group, the exoskeleton needs to provide complete support for STS action. For the latter group, on the other hand, exoskeletons should only compensate for the insufficient torque for STS action. Excessive support for this group of wearers may cause overreliance, which leads to further loss of muscle strength.

During an STS action, the hip, knee, and ankle are the three main joints that need to be actuated. Previous studies suggest that the knee joint is the main limiting joint for the STS action [15-17]. Exoskeletons can directly act on the knee joint to compensate for the lacked strength at knee joint, without interfering with other joints. Earlier knee exoskeleton have been shown to be beneficial in assisting STS motion [14].

Many existing STS exoskeletons in the literature use revolute pivot joints as their knee joints [12-14]. Human knee joints, on the other hand, have moving instantaneous centers (IC) due to the shape of the condyle and ligaments [18-20]. This mismatch between the exoskeleton and human biomechanics creates discomfort and increases metabolic consumption [21].

One solution to the problems above is to utilize an actuator with unique characteristics such as a Hydraulic Artificial Muscle (HAM) [22]. A HAM has very high force output at rest length, but weakens as the contraction ratio increases. This characteristic can be exploited as both a range of motion limiting mechanism and the provision of necessary torque outputs at corresponding knee joint angles without a control system.

In this study, a knee supporting device based on crossed four-bar linkage powered by HAM is proposed. Extensive research on the four-bar linkage has been done to emulate the knee ligaments and replicate the movement of the human knee joint. The Angled Bar is added to an existing four-bar linkage design to provide torque to the mechanism with considerations to the HAM's characteristics. The HAM provides high force density and back-drivability to the device, in addition to the unique force-contraction ratio relationship.

\section{Design}

The design of the device consists of two main parts: the HAM and the modified four-bar linkage.

\section{Hydraulic artificial muscle}

Construction of the HAM can simply be described as a rubber tube surrounded by a woven sleeve. When pressure is applied to the inner tube, it expands radially, widening the braiding angle of the sleeve, which creates contraction force in the axial direction. Both ends of the
HAM used in this research are sealed with aluminum fittings which fasten the rubber tube and the outer sleeve together, and provide an inlet/outlet port for hydraulic pressure [22]. A photograph of the HAM is shown in Fig. 1. The HAM can only generate force in the direction of contraction. The theoretical contraction force is described in [23] as shown below,

$$
f=\frac{\pi D^{2} P}{4}\left(3 \cos ^{2} \theta-1\right)
$$

where:

$$
\begin{aligned}
& f=\text { contraction force } \\
& \theta=\text { braiding angle } \\
& D=\text { diameter of tubing when } \theta=90^{\circ} \\
& P=\text { applied pressure }
\end{aligned}
$$

which can also be rewritten as a function of contraction, as

$$
f=\frac{\pi d_{0}^{2} P}{4} \frac{1}{\sin ^{2} \phi_{0}}\left(3(1-\varepsilon)^{2} \cos ^{2} \phi_{0}-1\right)
$$

where:

$$
\begin{aligned}
& d_{0}=\text { initial diameter } \\
& \phi_{0}=\text { initial braiding angle } \\
& \varepsilon=\text { contraction of the HAM }
\end{aligned}
$$

Comparisons between theoretical and experimental contraction force of the HAM is shown in Fig. 2. This model only considers hydraulic pressure inside the tube and contraction force created from the increased braiding angle, ignoring the thickness of rubber tube, elasticity of rubber tube and braiding sheath, and friction between surfaces of the components. Due to the reasons above, this model cannot explain why maximum contraction

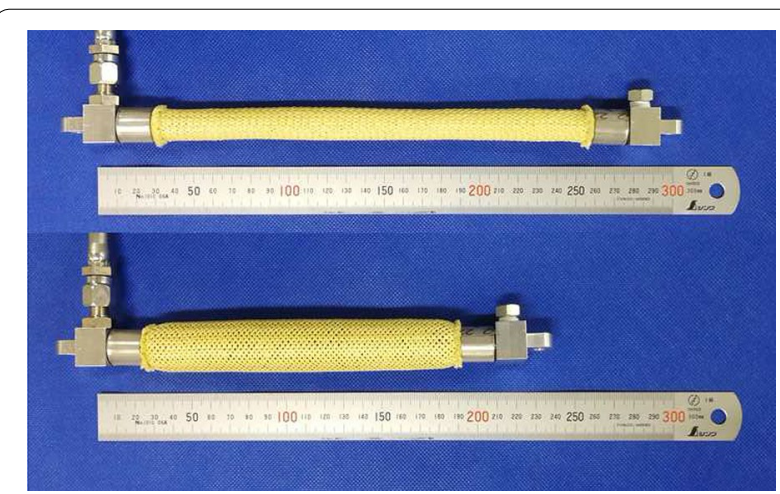

Fig. 1 Photo of the HAM used in this study in rest state and contracted state with $3 \mathrm{MPa}$ applied pressure 


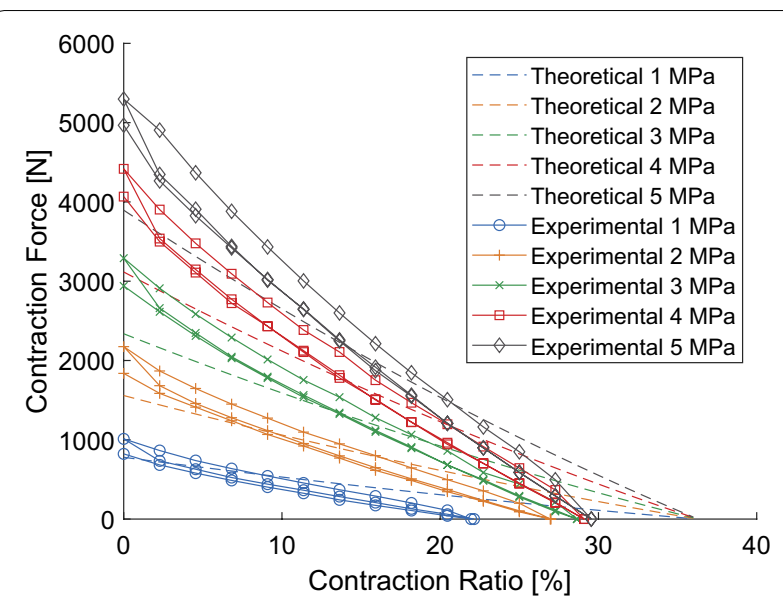

Fig. 2 Theoretical and experimental $F-\varepsilon$ relationship of the HAM used in this paper

ratio increases as the supplied pressure is increased. Finally, it also ignores hysteresis properties of the rubber, which results in different stress-strain curve during the loading and unloading of the HAM. The hysteresis loops occur due to Mullins effect, which is a mechanical property of rubbers [24].

The HAM has many advantages compared to traditional hydraulic actuators such as a hydraulic cylinder. First, it is lighter and has higher force density per mass and energy density per mass than other actuators, which are typically made from metal [22]. This is especially useful in wearable robot applications, where increased weight along the limbs creates higher inertia in addition to higher load from the weight. Secondly, it is inherently compliant due to its material being rubber. This is a beneficial characteristic for wearable robots and can be easily realized with a HAM without needing advanced control. Lastly, its force output depends on its stroke. This idea will be further discussed in the next paragraph.

Contraction force of the HAM depends on its contraction length as shown in Fig. 2. Its contraction force is highest at rest length, and gradually decreases as it contracts. Contracting the HAM beyond its neutral length quickly leads to buckling. This characteristic can be employed in applications where force requirement is not constant such as a STS support device. Forcecontraction ratio of the HAM can be matched with the torque requirement curve of the device. Furthermore, since HAM cannot contract beyond its minimum contraction length, it physically cannot actuate beyond the designed range of motion and serves as an intrinsic range of motion limiter.

The HAM used in this study can operate at up to 5 $\mathrm{MPa}$, in which it has a contraction force of $5.3 \mathrm{kN}$ and approximately $29.5 \%$ contraction ratio. However, the operating point of $3 \mathrm{MPa}$ was selected. Properties of the HAM used in this study are shown in Table 1.

\section{Modified four-bar linkage}

Various types of four-bar linkage knee mechanisms have been previously proposed with different design goals such as improving knee stability [25], replicating the shape of condyle [26], mimicking ligaments [27], or creating the desired instantaneous helical axis [28]. In this paper, a fourbar linkage based on design proposed in [26] is utilized.

Let point $\mathrm{A}$ be the origin of the coordinate system, with the knee joint angle defined as $0^{\circ}$ at full extension, and increases in the flexion direction. The configurations of the four-bar linkage are shown in Figs. 3, 4. The difference between the knee joint angle $\theta_{\text {knee }}$ and $\theta_{2}$ is equal to the initial angle of $\theta_{2}$, which is defined as $\theta_{i}$.

$$
\theta_{\mathrm{knee}}=\theta_{2}-\theta_{i}
$$

The angle $\theta_{1}$ as a function of $\theta_{2}$ can be found from a solution of Eq. 4.

\section{Table 1 Properties of the HAM used in this paper}

\begin{tabular}{lc}
\hline Parameter & Value \\
\hline Rest length & $220 \mathrm{~mm}$ \\
Maximum pressure & $5 \mathrm{MPa}$ \\
Maximum contraction force & $5.3 \mathrm{kN}$ \\
Maximum contraction ratio & $29.5 \%$ \\
Outer tubing diameter & $11 \mathrm{~mm}$ \\
Initial braiding angle & $25^{\circ}$ \\
Dry weight (including fittings) & $160 \mathrm{~g}$ \\
\hline
\end{tabular}

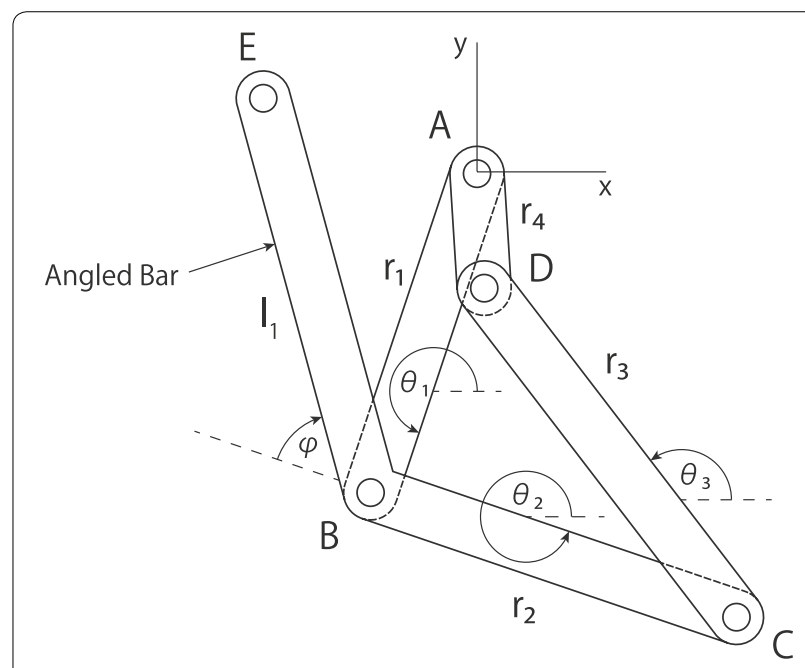

Fig. 3 Four-bar joint mechanism with Angled Bar at $\theta_{\text {knee }}=0^{\circ}$ 


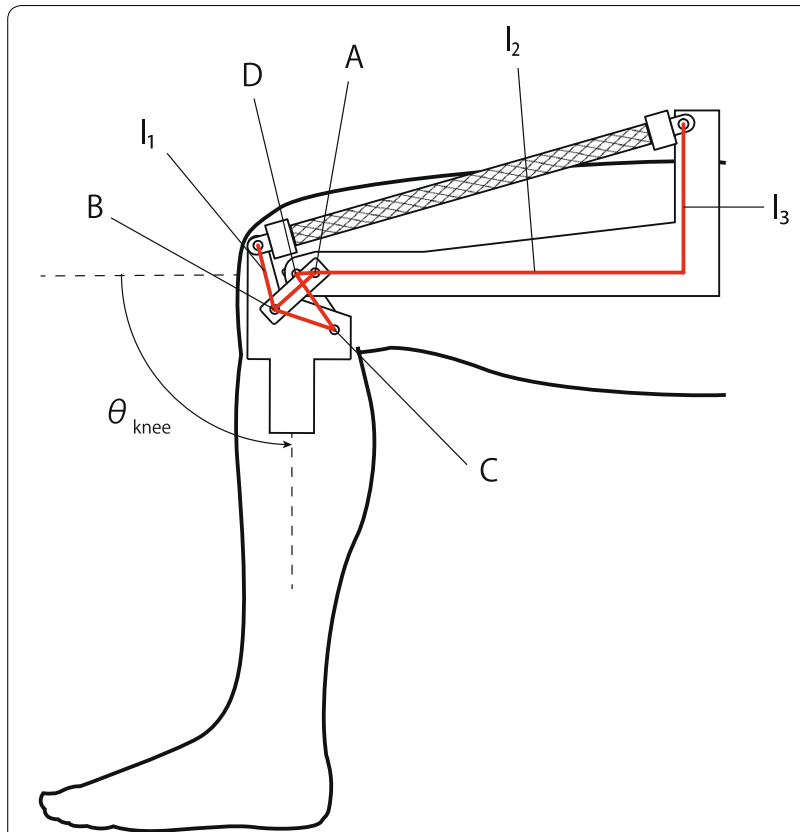

Fig. 4 Diagram of the developed device. Red lines represent four-bar linkage, as well as $I_{1}, l_{2}$ and $l_{3}$

$$
(P-Q) \tan ^{2}\left(\frac{\theta_{1}}{2}\right)-2 R \tan \left(\frac{\theta_{1}}{2}\right)+P+R=0
$$

where:

$$
\begin{aligned}
& r_{1}=\text { length of link } A B \\
& r_{2}=\text { length of link } B C \\
& r_{3}=\text { length of link } C D \\
& P=r_{3}^{2}-r_{1}^{2}-\left(x_{D}-r_{2} \cos \theta_{2}\right)^{2}-\left(y_{D}-r_{2} \sin \theta_{2}\right)^{2} \\
& Q=2 r_{1}\left(x_{D}-r_{2} \cos \theta_{2}\right) \\
& R=2 r_{1}\left(y_{D}-r_{2} \sin \theta_{2}\right)
\end{aligned}
$$

which will allow the position of point $B$, and subsequently, $E$, to be obtained.

The IC of link $B C$ is located at the intersection of link $A B$ and $C D$ [29]. Finally, the torque output can be calculated from the cross product of position vector and force vector.

Angled Bar $B E$ is extended from link $B C$ as shown in Fig. 3 to act as a lever and allow adjustments to the output torque curve with considerations to the force output of the HAM. From the diagram shown in 4, parameters that affect the performance of the device are $l_{1}, l_{2}, l_{3}$, and $\varphi$. The effects of each parameter is discussed below.

Since torque is defined as the cross product of position vector and force vector, the output torque can be increased by increasing the force vector's magnitude and position vector's length. Force can be maximized by increasing pressure or adding additional HAMs,

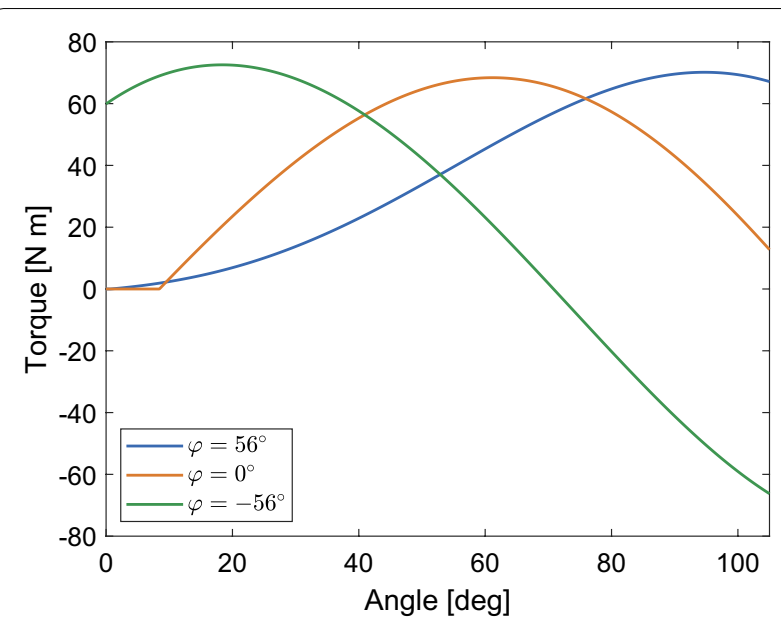

Fig. 5 Torque curve of the device with positive, zero, and negative $\varphi$ values

which can be easily be added infinitely so long as other constraints such as space, weight, or material strength allows. The position vector, which corresponds to the length of the angled bar $l_{1}$, however, is limited by stroke of the actuator and the desired motion range. Thus, both the length of HAM and link $l_{2}$ should both be maximized to increase the contraction distance. In the case that the length of HAM is already determined, $l_{2}$ should be adjusted according to the length of the HAM and the desired range of motion, which is determined by the link $l_{1}$ and $\varphi$.

The parameter $\varphi$ correlates to the shape of the output torque curve as illustrated in Fig. 5. Generally, negative $\varphi$ values lead to torque curve peaks at larger joint angles, while positive $\varphi$ values lead to opposite results. On the other hand, larger $l_{1}$ values lead to higher peaks in the torque curve, but also reduce motion range.

Finally, $l_{3}$ should be maximized to prevent HAM from moving over IC, which will generate torque in the opposite direction.

\section{Simulation and optimization}

In this section, simulations were used to obtain optimal parameters for the proposed device. First, an STS model was developed to find the required torque to be used as a target for optimization. Next, $l_{1}, l_{2}, l_{3}$, and $\varphi$ values that gave the most similar torque-angle graph as obtained from the STS model were selected.

\section{Sit-to-stand model}

To obtain the required torque as a function of knee joint angle, an STS model was created according to STS data in [17] and body parameters in [30,31], namely male body model with $73.0 \mathrm{~kg}$ weight and $1.741 \mathrm{~m}$ height was used. 
The model's arms were crossed over the chest. The headarm-trunk, thigh, and shank were assumed to be three rigid bodies as shown in Fig. 6 .

The model showed highest torque requirement at 72.49 $\mathrm{Nm}$ per leg at the beginning of the STS action, and lessened as the joint angle became larger. The model agrees with the literature, which reported peak $0.9 \mathrm{Nm} / \mathrm{kg}$ body mass per leg [7] or $1.17 \mathrm{Nm} \mathrm{kg}^{-1} \mathrm{~m}^{-1}$ with respect to body-weight times height [32], and maximum torque is reached at seatoff $[7,17,32,33]$. The resulting torque at ankle, knee, and hip is shown in Fig. 7.

\section{Artificial muscle model}

The HAM was modeled to have linear contraction force without hysteresis based on data obtained from experiments rather than theoretical force shown in Eq. 2. A least-squares regression was performed on the unloading contraction force curve to find the linear relation.

$$
y=\beta_{0}+\beta_{1} x+e
$$

where:

$$
\begin{aligned}
& \beta_{0}=y \text {-intercept, or the maximum contraction force } \\
& \beta_{1}=\text { regression coefficient } \\
& e=\text { error term }
\end{aligned}
$$

The maximum contraction ratio of the HAM is found at $\mathrm{x}$-intercept

$$
\varepsilon_{\max }=-\frac{\beta_{0}}{\beta_{1}}
$$

Stiffness beyond maximum contraction ratio was ignored due to unpredictably in buckling, and the force output was assumed to be zero. Finally, the force-contraction relationship of the HAM used during the design process is described by Eq. 7 .

$$
F(\varepsilon)= \begin{cases}\beta_{0}+\beta_{1} \varepsilon & \text { if } 0 \leq \varepsilon<\varepsilon_{\max } \\ 0 & \text { if } \varepsilon \geq \varepsilon_{\max }\end{cases}
$$

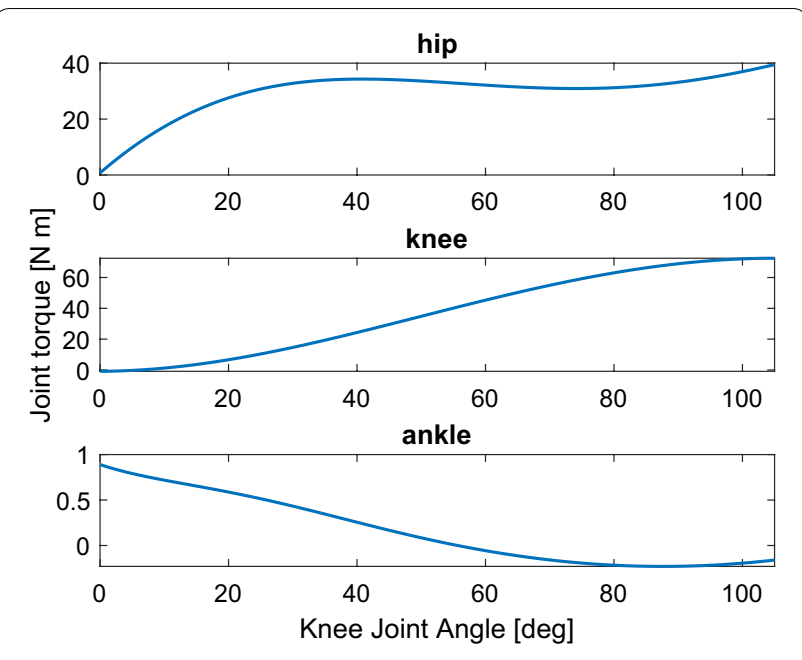

Fig. 7 Joint torque of hip, knee, and ankle joint during STS action

Contraction force beyond rest length was undefined. The force-contraction ratio relationship of the modeled HAM is shown in Fig. 8.

\section{Angled bar parameters}

The HAM used in this study has $220 \mathrm{~mm}$ rest length, and the parameters $l_{1}, l_{2}, l_{3}$, and $\varphi$ were adjusted accordingly. The HAM was specified not to exceed the rest length for safety reasons. The cost function utilized was root-meansquare error (RMSE), which was defined as

$$
R M S E=\sqrt{\frac{1}{n} \sum_{i=1}^{n}\left(T_{\text {req }, i}-T_{i}\right)^{2} .}
$$

where:

$$
\begin{aligned}
& n=\text { number of sampled points } \\
& T_{r e q, i}=\text { torque at knee joint shown in Fig. } 7 \\
& T_{i}=\text { torque generated using selected parameters }
\end{aligned}
$$

The best combination of parameters was found through exhaustive search. Every integer values of $l_{1}, l_{2}$ and $l_{3}$ within the interval $[0,100],[200,300]$, and $[0,100] \mathrm{mm}$
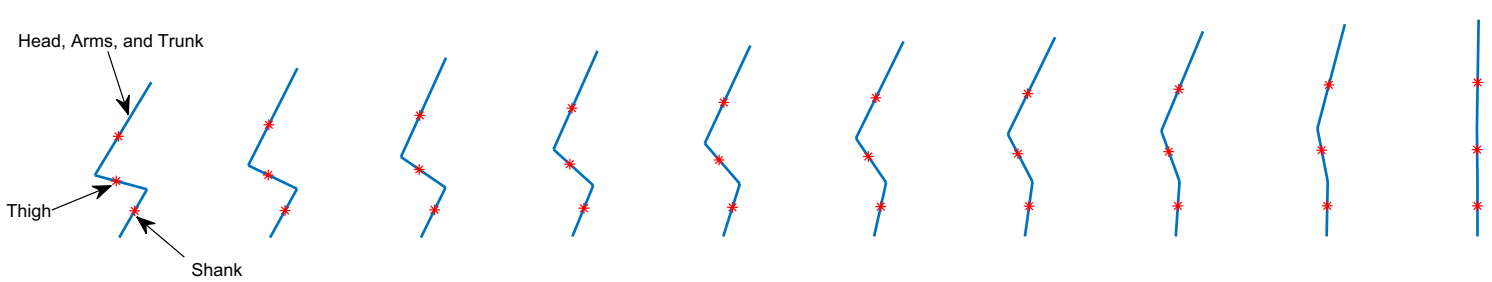

Fig. 6 STS model used in this study. Asterisks represent Center of Mass of each rigid body 


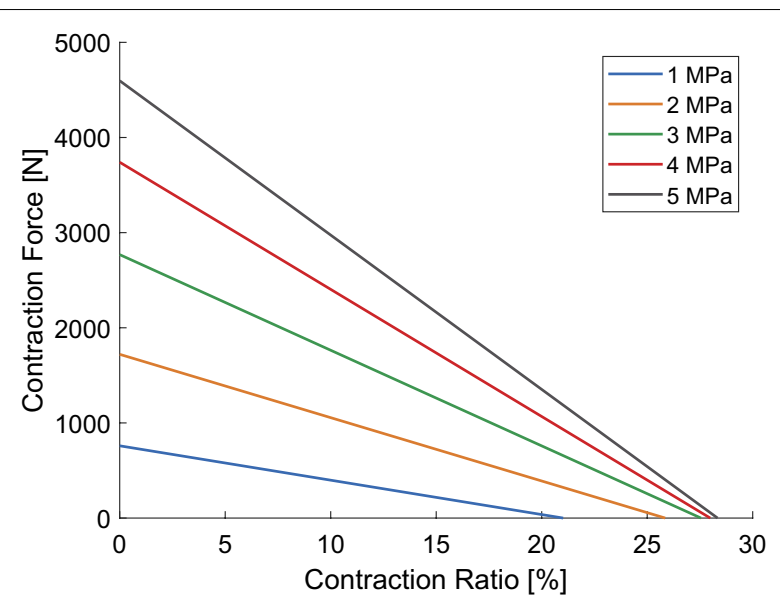

Fig. $8 F-\varepsilon$ relationship of the HAM used during the design process. Rubber hysteresis was not accounted for during the design process

respectively were used. The upper limit of $\varphi$ was found analytically and $\varphi$ between [0 71] degree were utilized.

Simulations were done at $2 \mathrm{MPa}, 3 \mathrm{MPa}, 4 \mathrm{MPa}$, and 5 $\mathrm{MPa}$. Results above $3 \mathrm{MPa}$ showed no significant differences as illustrated in Fig. 9, thus $3 \mathrm{MPa}$ was selected for better safety and lower pump requirements.

Moreover, simulation results showed improved performance over a hydraulic cylinder with similar design optimization methods as shown in Fig. 10. Two cylinders were analyzed for comparisons: a hydraulic cylinder with $11 \mathrm{~mm}$ piston diameter, which is the same size with HAM's initial diameter, operated at $3 \mathrm{MPa}$; and a hydraulic cylinder with $2769 \mathrm{~N}$ contraction force, which is HAM's maximum contraction force at $3 \mathrm{MPa}$. The cylinders were modeled to have constant contraction force

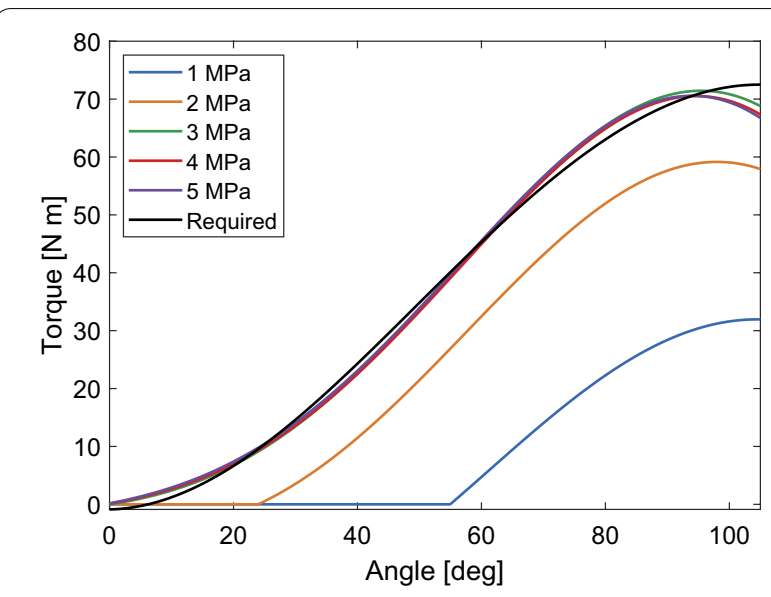

Fig. 9 Simulation results of optimal torque curve at different HAM pressures obtained through optimization

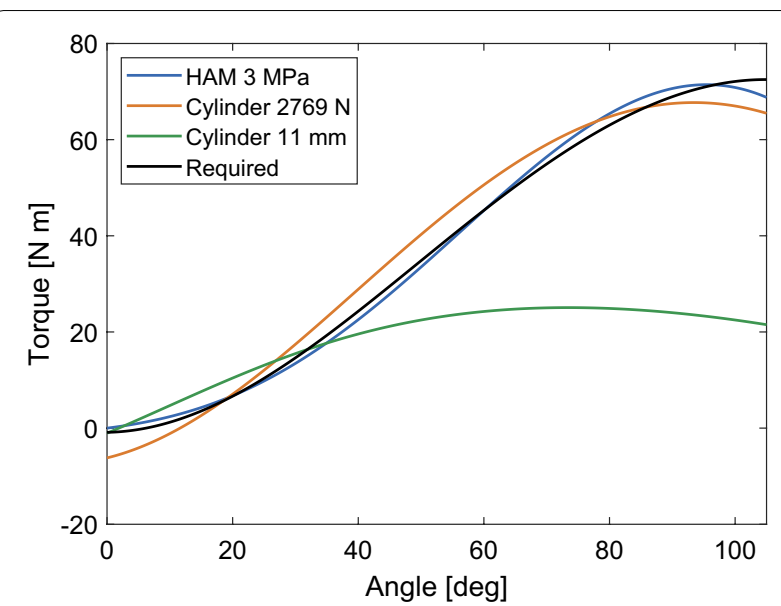

Fig. 10 Simulation results of generated torque curve using hydraulic cylinders as actuators. Similar optimization method was utilized

throughout their strokes. The piston rod was ignored for the $11 \mathrm{~mm}$ diameter cylinder.

The torque curve obtained using a similar-sized cylinder shows much lower torque output. On the other hand, the torque curve obtained using the cylinder with similar contraction force has larger error, lower peak torque, and negative torque output at low knee joint angle. The negative joint torque output may hinder the STS performance and thus is undesired. An additional control system is required to manipulate the hydraulic pressure and obtain the appropriate torque curve.

The superior performance of the HAM can be achieved due to its high contraction force. To obtain a peak torque similar to the HAM, a much larger cylinder or $l_{1}$ values is required. In addition, HAMs' force-contraction ratio relationship can be exploited, as opposed to cylinders where force is constant regardless of stroke length. This means that the HAM and optimized linkage design realize the required torque-angle properties without complicated hydraulic pressure control but only by keeping constant pressure. This makes the hydraulic control system very simple and practical.

\section{Experiments}

\section{Output torque measurement}

A photograph of the developed device is shown in Fig. 11. Experiments were carried out to determine the torque output of the device. The shank part of the knee joint was extended using an aluminum bar. The device was clamped vertically at the thigh part, and $3 \mathrm{MPa}$ hydraulic pressure was supplied to the device. Force was applied to the end of the aluminum bar by hand by pressing the force gauge against the bar. To measure the loading torque curve, force was applied to the bar from 
neutral (extended) position until the shank part moved to the measuring angle. For the unloading torque curve, the shank part was first moved to the maximum flexion angle of $105^{\circ}$, then the applied force was gradually reduced and force was measured when the shank part reached the measuring angle.

Output torque of the device was calculated by applied force multiplied by distance to IC plus torque created by weight of the extended bar. Force was measured using a force gauge (FGP-50, SHIMPO), and the angle was measured by digital angle gauge (WR300, WIXEY). Pressure was supplied from a hydraulic unit (YU-65912, HI-PLAN) controlled with a hydraulic valve (BSDFA03P-315, WANDFLUH). Pressure was measured using a digital pressure transmitter (8252.83.2517, TRAFAG). The experimental setup is shown in Fig. 12.

The output torque shows low output torque at low knee joint angle, and increases as the angle becomes larger, similar to the required output curve as shown in Fig. 13. The device shows a different torque curve during knee extension and flexion due to the hysteresis characteristic of the HAM, which has different contraction forces during loading and unloading. During STS, the knee joint moves in extension direction (i.e., reducing knee joint angle) therefore torque curve in the extension direction should be evaluated to correctly interpret the results.

Experimental results in the extension direction agreed well with the expected results up to $100^{\circ}$. The RMSE of expected results and experimental knee extension results were $1.98 \mathrm{Nm}$ and $13.01 \mathrm{Nm}$, respectively. If the $105^{\circ}$

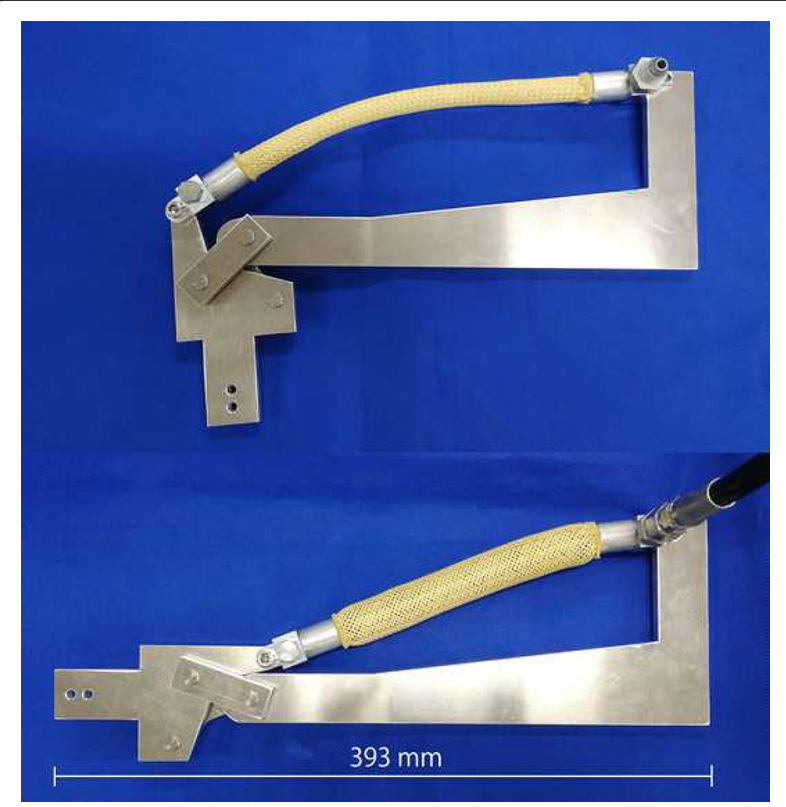

Fig. 11 Photo of the developed device

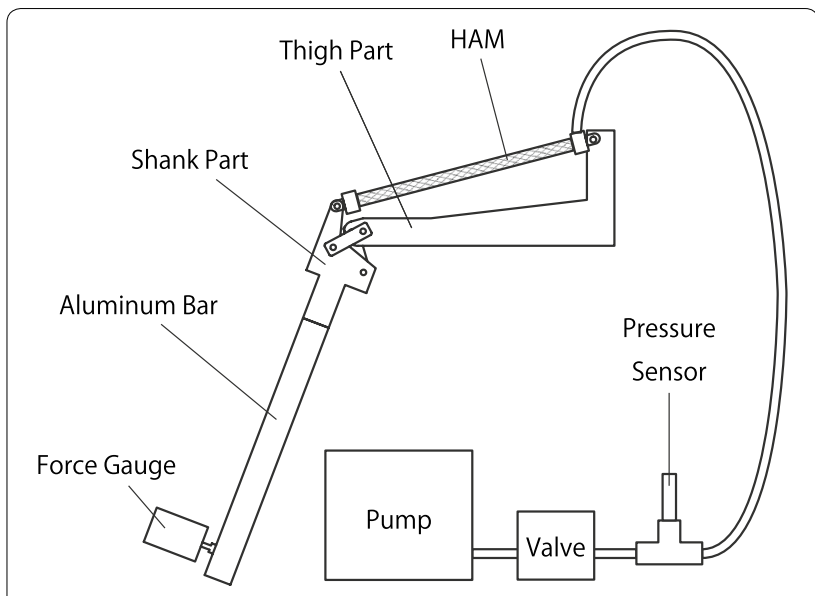

Fig. 12 Experimental setup

point is discarded, the RMSE would reduce to $5.048 \mathrm{Nm}$. We believe this was caused by non-linear force-contraction curve which was ignored during the design process, and mechanical deformations of the links.

\section{Support evaluation}

To validate the reduction of muscle activity and verify the degree of support provided by the device during STS, surface electromyography (EMG) of a healthy participant was measured. The subject was a 24-year-old male, with $1.65 \mathrm{~m}$ body height and $56.4 \mathrm{~kg}$ body mass. An aluminum bar, 3D printed braces, and straps were added to the device to attach the device to the leg of the user. The device was worn only on the right leg as shown in Fig. 14. Hydraulic pressure was reduced to $2.30 \mathrm{MPa}$ in proportion to the participant's body mass.

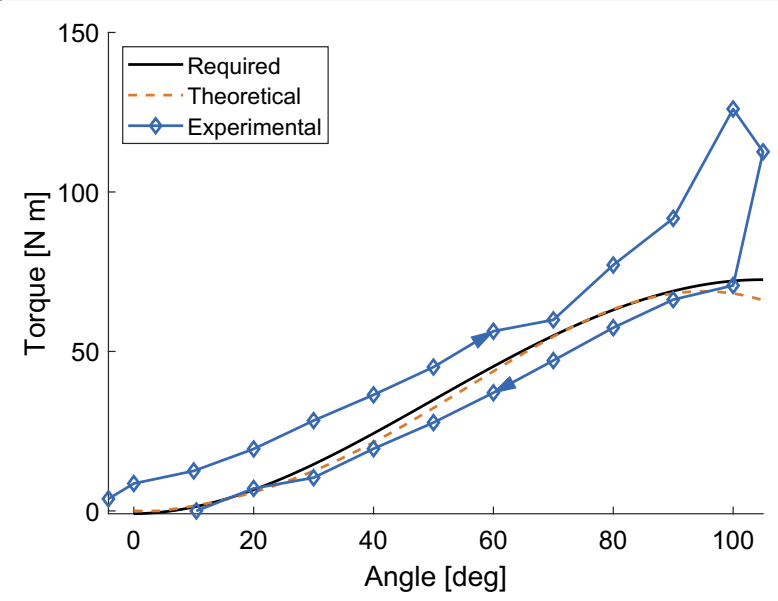

Fig. 13 Torque output results of the device 


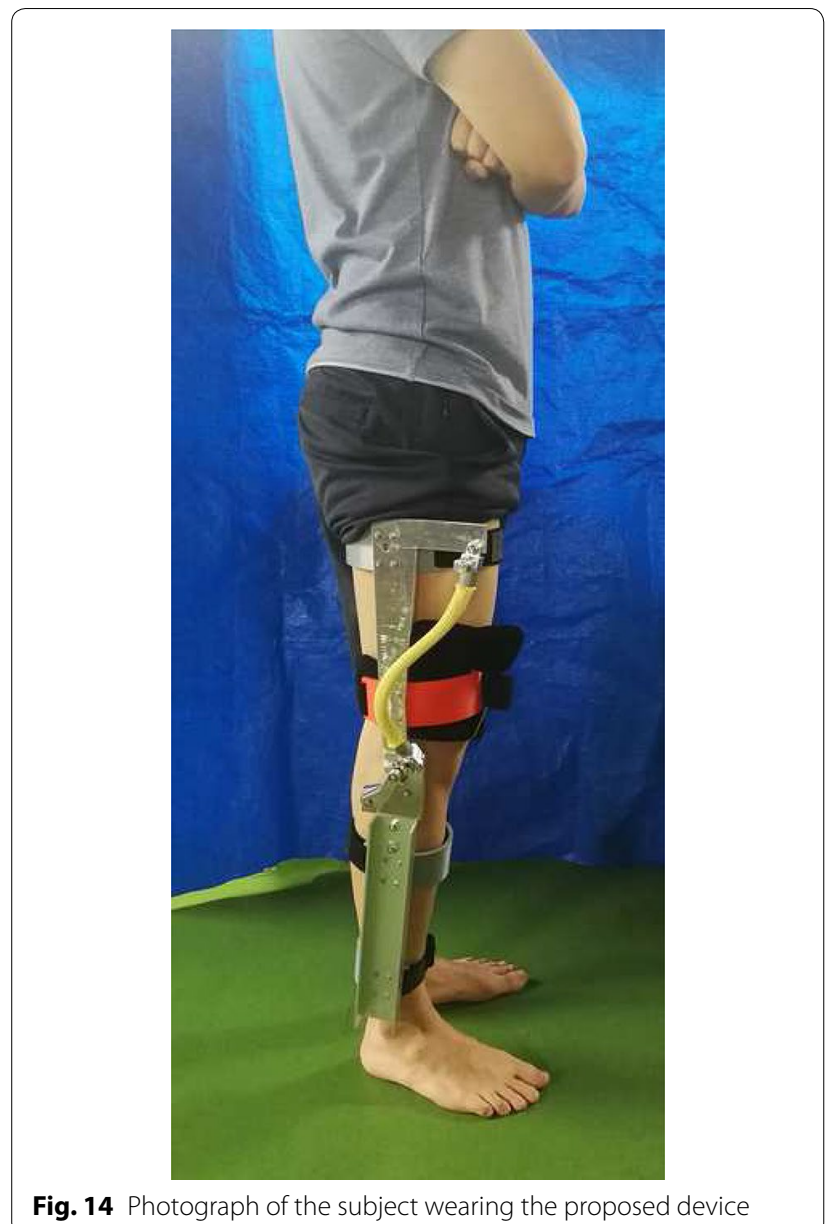

The subject performed three trials of the experiment while wearing the device, each time with and without assist from the device. One trial of experiment consists of sitting in normal position for $60 \mathrm{~s}$, sitting in seatoff position for $10 \mathrm{~s}$, performing STS at natural speed, and stand for $10 \mathrm{~s}$. For the active trial, the device was activated at the start of STS. The subject's arms were folded across the chest for the whole duration of the experiment. The subject tried to keep the ground reaction force on both feet equal.

The EMG signal on the right Rectus femoris was measured using a surface electromyography device (FREEEMG 1000, BTS Bioengineering). The signal was sampled at $1 \mathrm{kHz}$, and processed with sliding window root mean square of 200 samples windows length. The signals were normalized to the STS duration, then the averages between the three trials were calculated.

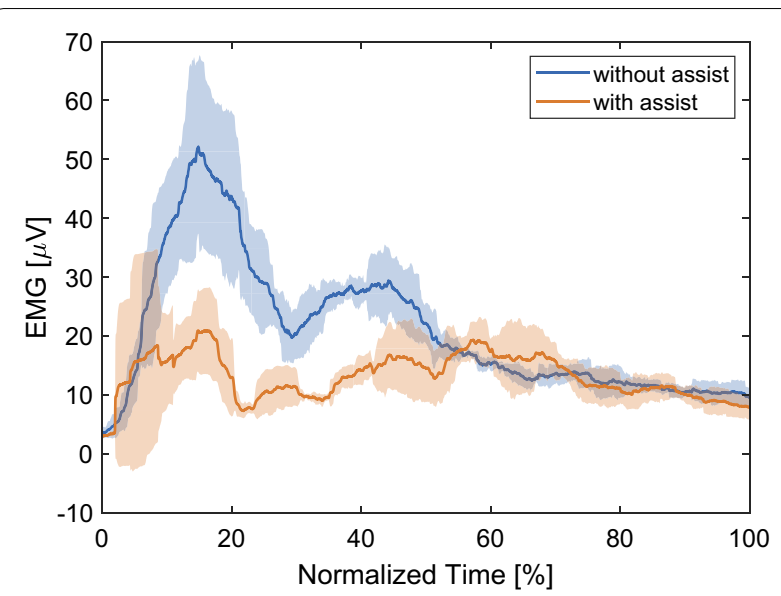

Fig. 15 Processed EMG signal during STS action with and without assist of the device. The shaded regions illustrate the region of \pm 1 standard deviation around the average

The results show significant reduction in muscle activity with support from the device as shown in Fig. 15. The maximum processed EMG signal with and without assist were 52.10 and $20.93 \mu \mathrm{V}$, respectively.

\section{Discussion}

In actual application of the device, every individual has different physical properties such as body mass, height, leg length, and muscle strength. These different properties make the required torque curve for every individual unique. While this may be true, the output torque of the device can also be adjusted by changing the applied hydraulic pressure. The highest torque requirement for STS is at seat-off, which is approximately proportional to body mass. Following this approximation, the device at $5 \mathrm{MPa}$ applied pressure would be able to support a fully paraplegic subject up to $109 \mathrm{~kg}$ body mass. For users who have limited muscle strength, the device's torque output can be lowered to match the required degree of support by reducing the hydraulic pressure.

The device should be worn firmly to facilitate transfer of torque between the exoskeleton and wearer's knee. The braces and straps should be sufficiently tight and able to adapt to different leg shapes without being uncomfortable to the wearer. The thigh and shank part should also be able to be configured for different leg alignments. These issues will be studied in the future.

To provide hydraulic pressure for the device, a hydraulic power supply is required. A small pump unit can be utilized. For example, a supply unit proposed in 
[34] has a total weight of $2.2 \mathrm{~kg}$ and can provide up to $12 \mathrm{MPa}$ pressure, which is much higher than the pressure requirement of this study. Another solution is to utilize a follower robot to provide mobile hydraulic supply such as those proposed in [35].

\section{Conclusion}

In this paper, a knee support device was developed using four-bar linkage joint mechanism and HAM. A STS model was created to obtain torque at knee, hip, and ankle. The Angled Bar was proposed to adjust the contraction force of HAM to the four-bar joint to generate a differing knee joint torque at the corresponding angular position. The required torque curve was obtained only by applying constant $3 \mathrm{MPa}$ pressure without pressure adjustments or the use of a complicated control system. This allows a simple hydraulic controller to be utilized, which reduces the complexity of the system and improves practicality. Finally, the applicability of the developed device was validated through significant reduction of muscle activity on a healthy participant, which was measured using EMG. Application of HAM led to a simpler device with a superior performance compared to equivalent hydraulic cylinders, in addition to lower weight and passive backdrivability.

\section{Acknowledgements}

Not applicable.

\section{Authors' contributions}

All authors read and approved the final manuscript.

\section{Funding}

This work was supported by JSPS KAKENHI Grant-in-Aid for Scientific Research (A) under Grant Number JP18H03760.

\section{Availability of data and materials}

Not applicable.

\section{Competing interests}

The authors declare that they have no competing interests.

\section{Author details}

1 Department of Mechanical Engineering, Tokyo Institute of Technology, 2-12-1 Ookayama, Meguro-ku, Tokyo 152-8550, Japan. ${ }^{2}$ Advanced Technology and Design Creating Dliv., Bridgestone Corporation, 3-1-1 Ogawahigashi-cho, Kodaira-shi, Tokyo 187-0031, Japan.

Received: 26 November 2019 Accepted: 12 March 2020 Published online: 27 March 2020

\section{References}

1. Dall PM, Kerr A (2010) Frequency of the sit to stand task: an observational study of free-living adults. Appl Ergon 41(1):58-61. https://doi. org/10.1016/J.APERG0.2009.04.005

2. Grant PM, Dall PM, Kerr A (2011) Daily and hourly frequency of the sit to stand movement in older adults: a comparison of day hospital, rehabilitation ward and community living groups. Aging Clin Exp Res 23(5-6):437-44
3. Jensen J, Lundin-Olsson L, Nyberg L, Gustafson Y (2002) Falls among frail older people in residential care. Scand J Public Health 30(1):54-61. https ://doi.org/10.1177/14034948020300011201

4. Rapp K, Becker C, Cameron ID, König H-H, Büchele G (2012) Epidemiology of falls in residential aged care: analysis of nore than 70,000 falls from residents of Bavarian nursing homes. J Am Med Dir Assoc 13(2):187-11876. https://doi.org/10.1016/J.JAMDA.2011.06.011

5. Fried LP, Ferrucci L, Darer J, Williamson JD, Anderson G (2004) Untangling the concepts of disability, frailty, and comorbidity: implications for improved targeting and care. J Gerontol Ser A: Biol Sci Med Sci 59(3):255-263. https://doi.org/10.1093/gerona/59.3.m255

6. Rubenstein LZ (2006) Clinical risk assessment, interventions and services falls in older people: epidemiology, risk factors and strategies for prevention. Age Ageing. https://doi.org/10.1093/ageing/afl084

7. Kotake T, Dohi N, Kajiwara T, Sumi N, Koyama Y, Miura T (1993) An analysis of sit-to-stand movements. Archi Phys Med Rehabil 74(10):1095-1099. https://doi.org/10.1016/0003-9993(93)90068-L

8. Schenkman M, Hughes MA, Samsa G, Studenski S (1996) The relative importance of strength and balance in chair rise by functionally impaired older individuals. J A Geriatr Soc 44(12):1441-1446. https://doi. org/10.1111/j.1532-5415.1996.tb04068.x

9. Kamnik R, Bajd T (2004) Standing-up robot: an assistive rehabilitative device for training and assessment. J Med Eng Technol 28(2):74-80. https ://doi.org/10.1080/0309190032000112306

10. Fraiszudeen A, Yeow CH (2016) Soft robotic sit-To-stand trainer seat. In: Proceedings of the IEEE RAS and EMBS International Conference on biomedical robotics and biomechatronics, vol. 2016, p 673-679. IEEE Computer Society, UTown, Singapore. https://doi.org/10.1109/BIORO B.2016.7523703

11. Scaletta T, Komada S, Member Roberto Oboe S (2016) Development of a human assistive robot to support hip joint movement during sit-tostand using non-linear springs. IEEJ J Ind Appli 5(3):261-266. https://doi. org/10.1541/ieejjia.5.261

12. Tsukahara A, Kawanishi R, Hasegawa Y, Sankai Y (2010) Sit-to-stand and stand-to-sit transfer support for complete paraplegic patients with Robot Suit HAL. Adv Robotics 24:1615-1638. https://doi.org/10.1163/01691 $8610 \times 512622$

13. Huo W, Mohammed S, Amirat Y, Kong K (2016) Active impedance control of a lower limb exoskeleton to assist sit-to-stand movement. Proceedings - IEEE international conference on robotics and automation 2016. p 3530-3536. https://doi.org/10.1109/ICRA.2016.7487534

14. Shepherd MK, Rouse EJ (2017) Design and validation of a Torque-Controllable knee exoskeleton for sit-to-stand assistance. IEEE/ASME Trans Mechatron 22(4):1695-1704. https://doi.org/10.1109/TMECH.2017.27045 21

15. Hughes MA, Myers BS, Schenkman ML (1996) The role of strength in rising from a chair in the functionally impaired elderly. J Biomech 29(12):1509-1513. https://doi.org/10.1016/S0021-9290(96)80001-7

16. Corrigan D, Bohannon RW (2001) Relationship between knee extension force and stand-up performance in community-dwelling elderly women. Arch Phys Med Rehabil 82(12):1666-1672. https://doi.org/10.1053/ apmr.2001.26811

17. Yoshioka S, Nagano A, Himeno R, Fukashiro S (2007) Computation of the kinematics and the minimum peak joint moments of sit-to-stand movements. BioMed Eng Online 6(1):26. https://doi. org/10.1186/1475-925X-6-26

18. Hollman JH, Deusinger RH, Dillen LRV, Matava MJ (2002) Knee joint movements in subjects without knee pathology and subjects with injured anterior cruciate ligaments. Phys Ther 82(10):960-972. https://doi. org/10.1093/ptj/82.10.960

19. Hollman JH, Deusinger RH, Van Dillen LR, Matava MJ (2003) Gender differences in surface rolling and gliding kinematics of the knee. Clin Orthop Relat Res 413:208-221. https://doi.org/10.1097/01.blo.0000072902.36018 fe

20. Koo S, Andriacchi TP (2008) The knee joint center of rotation is predominantly on the lateral side during normal walking. J Biomech 41(6):12691273. https://doi.org/10.1016/J.JBIOMECH.2008.01.013

21. Huo W, Mohammed S, Moreno JC, Amirat Y (2016) Lower limb wearable robots for assistance and rehabilitation: a state of the art. IEEE Syst J 10(3):1068-1081. https://doi.org/10.1109/JSYST.2014.2351491 
22. Morita R, Nabae H, Endo G, Suzumori K (2018) A proposal of a new rotational-compliant joint with oil-hydraulic McKibben artificial muscles. Adv Robotics 32(9):511-523. https://doi.org/10.1080/01691864.2018.1464946

23. Schulte FH (1961) The Characteristics of the McKibben artificial muscle. In: The application of external power in prosthetics and orthotics, $\mathrm{P}$ 94-115. National Academy of Sciences-National Research Council, Washington.

24. Kurumaya S, Nabae H, Endo G, Suzumori K (2017) Design of thin McKibben muscle and multifilament structure. Sens Actuators A 261:66-74. https://doi.org/10.1016/j.sna.2017.04.047

25. Radcliffe CW (1994) Four-bar linkage prosthetic knee mechanisms: kinematics, alignment and prescription criteria. Prosthet Orthot Int 18(3):159-173. https://doi.org/10.3109/03093649409164401

26. Karami M, Maurice G, Andre JM (2004) A model of exo-prosthesis of the knee optimized with respect to the physiological motion of condyles. ITBM-RBM 25(3):176-184. https://doi.org/10.1016/J.RBMRET.2004.03.003

27. Zavatsky AB, O'Connor JJ (1992) A model of human knee ligaments in the sagittal plane: part 2: fibre recruitment under load. Proc Inst Mech Eng Part H: J Eng Med 206(3):135-145. https://doi.org/10.1243/ PIME PROC 199220628102

28. Bertomeu JMB, Lois JMB, Guillem RB, Del Pozo ÁP, Lacuesta J, Mollà CG, Luna PV, Pastor JP (2007) Development of a hinge compatible with the kinematics of the knee joint. Prosthet Orthot Int 31(4):371-383. https:// doi.org/10.1080/03093640601095842

29. Weisstein EW. Line-line intersection. Wolfram Research, Inc. http://mathw orld.wolfram.com/Line-Linelntersection.html Accessed 13 Nov 2019

30. Zatsiorsky VM (2002) Kinetics of Human Motion. Human Kinetics, Champaign
31. De Leva P (1996) Adjustments to zatsiorsky-seluyanov's segment inertia parameters. J Biomech 29(9):1223-1230. https://doi.org/10.1016/00219290(95)00178-6

32. Mak MKY, Levin O, Mizrahi J, Hui-Chan CWY (2003) Joint torques during sit-to-stand in healthy subjects and people with Parkinson's disease. Clin Biomech 18(3):197-206. https://doi.org/10.1016/S0268-0033(02)00191-2

33. Kamali K, Akbari AA, Akbarzadeh A (2016) Trajectory generation and control of a knee exoskeleton based on dynamic movement primitives for sit-to-stand assistance. Adv Robotics 30(13):846-860. https://doi. org/10.1080/01691864.2016.1154800

34. Nath JD, Durfee WK (2017) Optimization and design principles of a minimal-weight, wearable hydraulic power supply. In: ASME 2017 Dynamic Systems and Control Conference, DSCC 2017, vol. 1. American Society of Mechanical Engineers, Tysons. https://doi.org/10.1115/DSCC2017-5046

35. Tani A, Endo G, Fukushima EF, Hirose S, Iribe M, Takubo T (2011) Study on a practical robotic follower to support home oxygen therapy patientsdevelopment and control of a mobile platform. In: IEEE/RSJ International conference on intelligent robots and systems, pp 2423-2429. Institute of Electrical and Electronics Engineers (IEEE), San Francisco. https://doi. org/10.1109/iros.2011.6094633

\section{Publisher's Note}

Springer Nature remains neutral with regard to jurisdictional claims in published maps and institutional affiliations.

\section{Submit your manuscript to a SpringerOpen ${ }^{\odot}$ journal and benefit from:}

- Convenient online submission

- Rigorous peer review

- Open access: articles freely available online

- High visibility within the field

- Retaining the copyright to your article

Submit your next manuscript at $\mathbf{s p r i n g e r o p e n . c o m ~}$ 